DOI: $10.3901 / J M E .2021 .20 .133$

\title{
氢化物对锆合金薄板焊缝断裂行为的影响
}

\author{
王 博 ${ }^{1}$ 包 陈 $^{1}$ 魏连峰 $^{2}$ 何广伟 $^{1}$ \\ (1. 西南交通大学力学与工程学院 成都 610031;
}

2. 中国核动力研究设计院反应堆燃料与材料重点实验室 成都 610041)

\begin{abstract}
摘要: 铅合金被广泛应用于反应堆的燃料管包壳材料, 当服役过程中铅合金的力学性能改变时, 对其力学性能完整性的评估 至关重要。基于小尺寸三点弯曲试样, 建立锆合金薄板焊缝的断裂韧度测试方法, 完成腐蚀渗氢后锆合金焊缝在室温和 $360{ }^{\circ} \mathrm{C}$ 下准静态断裂㓞度试验, 分析氢腐蚀和温度对铅合金焊缝断裂性能的影响。研究结果表明, 氢腐蚀和温度均能对铅合金焊缝 断裂性能产生显著影响, 由于高温条件下聚集在裂尖附近的氢化物溶解, 使得 $360{ }^{\circ} \mathrm{C}$ 下钻合金渗氢焊缝断裂韧度较室温下的 断裂韧度有显著提升。
\end{abstract}

关键词: 铅合金; 焊缝; 渗氢腐蚀; 小试样; 断裂韧度

中图分类号: $\mathrm{O} 346$

\section{Effect of Hydride on Fracture Behavior of Zirconium Alloy Platy Welds}

\author{
WANG Bo ${ }^{1}$ BAO Chen ${ }^{1}$ WEI Lianfeng ${ }^{2} \quad$ HE Guangwei $^{1}$ \\ (1. School of Mechanics and Engineering, Southwest Jiaotong University, Chengdu 610031; \\ 2. Science and Technology on Reactor Fuel and Material Laboratory, \\ Nuclear Power Institute of China, Chengdu 610041)
}

\begin{abstract}
Zirconium alloys are widely used in reactor fuel tube cladding, it is very important to evaluate the mechanical integrity of zirconium alloys when their mechanical properties change in service. Fracture toughness test method of small zirconium alloy platy welds was established based on small size three-point bending sample. The quasi-static fracture toughness tests of hydrogenated zirconium alloy welds at room temperature and $360{ }^{\circ} \mathrm{C}$ were carried out. The influence of hydrogen corrosion and temperature on fracture performance of zirconium alloy welds was analyzed. The results show that hydrogen corrosion and temperature can significantly affect the fracture properties of zirconium alloy welds. The fracture toughness of hydrogenated zirconium alloy welds at $360{ }^{\circ} \mathrm{C}$ enhances evidently compared with that at room temperature because of the dissolution of the hydrides distributed around crack tip.
\end{abstract}

Key words: zirconium alloy; weld; hydrogen corrosion; small sample; fracture toughness

\section{0 前言}

锆合金是一种新型的核反应堆燃料的结构包壳 材料, 具有较小的热中子吸收截面和优良的力学性 能。在反应堆运行的过程中, 铅合金会发生吸氢现 象 $^{[1]}$, 且吸收的氢大部分以氢化物的形式析出 ${ }^{[2-3]}$, 氢化物的存在对锆合金的力学性能有显著的影响, 如塑性降低, 强度提高等 ${ }^{[4]}$ 。而对氢化物本身的力 学性能, 存在不同的认识。一些研究认为氢化物是

20200420 收到初稿, 20210305 收到修改稿
完全脆性的 ${ }^{[5]}$, 另一些则认为氢化物能随基体产生 较大的塑性变形 ${ }^{[6]}$ 。

近年来, 针对锆合金的研究集中在拉伸, 疲劳, 氢致延迟开裂等方面。刘肖等 ${ }^{[7]}$ 研究发现, 锆合金 包壳管具有优良的抗裂性能, 氢含量低于 $200 \mathrm{mg} / \mathrm{kg}$ 时, 其对包壳管断裂韧度的影响较小; 当氢含量达 到 $290 \mathrm{mg} / \mathrm{kg}$ 时, 包壳管的断裂韧度显著提高 ${ }^{[7]}$ 。贾 琦等 ${ }^{[8]}$ 研究表明, 在等幅应变过程中, $\mathrm{Zr}-4$ 锆合金 表现为循环硬化，应变幅越低，硬化现象越明显; 孙超等 ${ }^{[9]}$ 研究发现 N18 锆合金发生氢致延迟开裂的 临界温度介于相同氢含量下溶解固溶温度与析出固 
溶温度之间。HSU 等 ${ }^{[10]}$ 发现, Zr-4 合金在室温 $\left(25^{\circ} \mathrm{C}\right)$ 时的径向氢化物会促进裂纹的扩展, 并降低 断裂韧度。MIN 等 ${ }^{[11]}$ 研究认为, 在含有径向氢化物 的包壳管材中微裂纹会在径向氢化物处形成, 并沿 着互相连接的径向氢化物生长, 最终导致包壳管材 的脆性断裂。TSENG 等 ${ }^{[12]}$ 在模拟氢化物对包壳材料 裂纹扩展的影响时, 发现径向氢化物对包壳材料的 裂纹扩展有显著影响。有关锆合金薄板焊缝断裂行 为的研究少见文献报道。由于本文所用的锆合金薄 板焊缝厚度仅为 $3.5 \mathrm{~mm}$, 传统的紧凑拉伸(Compact tension, CT)、三点弯曲(Single edge notch bending, SEB)等标准构型试样无法用于锆合金薄板焊缝的 断裂韧度测试。

本文采用小尺寸试样完成了锆合金薄板未渗氢 焊缝与渗氢焊缝在室温和 $360{ }^{\circ} \mathrm{C}$ 下断裂韧度试验, 研究了氢腐蚀和温度对锆合金薄板焊缝断裂韧度的 影响。

\section{1 材料与试验}

试验材料为 Zr-Sn-Nb 锆合金, 其化学成分为如 表 1 所示。

表 $1 \mathrm{Zr}-\mathrm{Sn}-\mathrm{Nb}$ 锆合金化学成分(质量分数) $\%$

\begin{tabular}{cccc}
\hline $\mathrm{Nb}$ & $\mathrm{Fe}$ & $\mathrm{Cr}$ & $\mathrm{O}$ \\
\hline $0.2 \sim 0.35$ & $0.3 \sim 0.4$ & $0.05 \sim 0.1$ & $0.09 \sim 0.12$ \\
\hline
\end{tabular}

锆合金焊缝采用电子束焊接工艺制成。电子束 焊接使用极致密的高速电子流打到被焊金属的接缝 上, 使其加热, 熔化金属, 冷却形成焊缝。为了模 拟某结构, 在锆合金板上设计一缺口, 然后采用电 子束焊接工艺进行焊接。图 1 为渗氢锆合金焊接板, 尺寸约为 $80 \mathrm{~mm} \times 50 \mathrm{~mm} \times 3.5 \mathrm{~mm}$, 每块锆合金板通 过电子束焊接方法焊接 3 条焊缝, 焊缝尺寸如图 $1 b$ 所示。

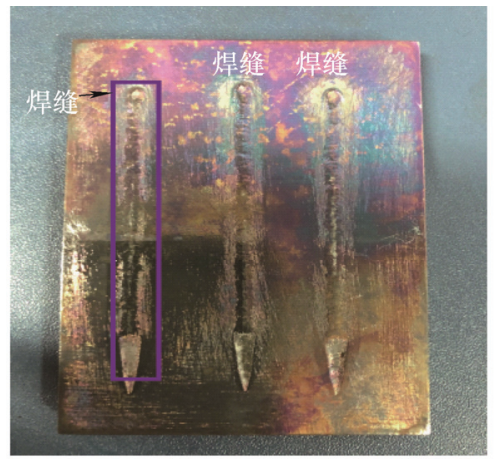

(a) 渗氢焊接板实物图

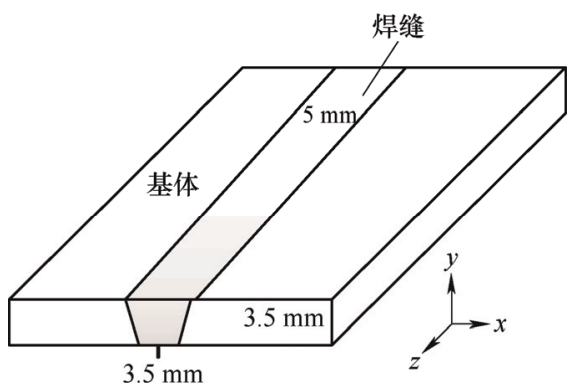

(b) 渗氢焊接板焊缝示意图

图 1 渗氢焊接板

采用高压釜渗氢方法, 将锆合金焊接板置于 $360{ }^{\circ} \mathrm{C}$ 碱性水中腐蚀 500 天。图 2 为高压釜腐蚀渗 氢后焊缝的金相照片。可以看到，所取锆合金焊板 存在未焊透现象, 焊缝在腐蚀后产生了一定数量的 氢化物。经分析，腐蚀后的锆合金焊缝氢浓度为 $0.035 \%$ 。 $\mathrm{Zr}$ 合金在吸氢过程会产生氢化物, 氢化物

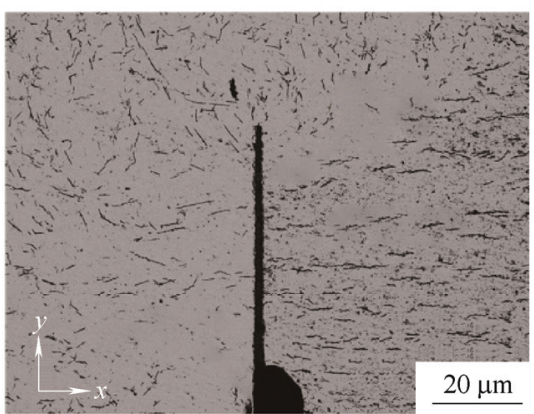

(a) 整体氢化物分布图

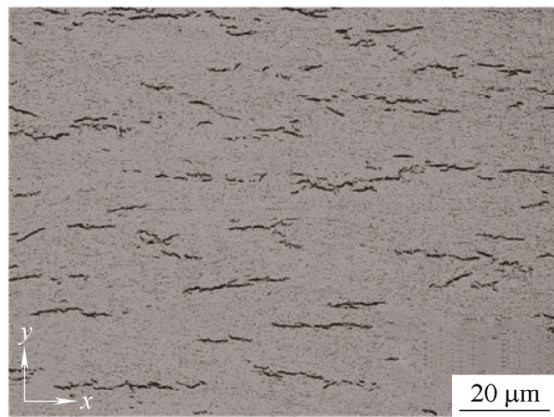

(b) 母材氢化物分布图

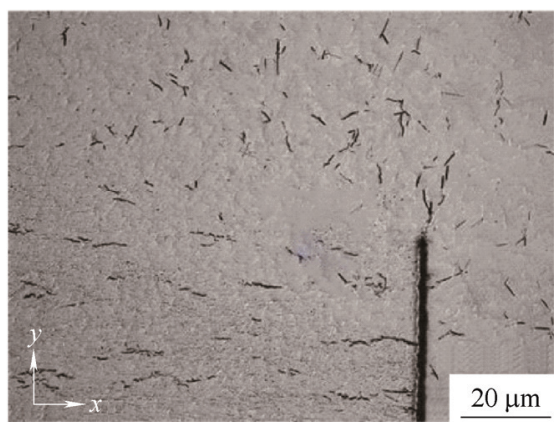

(c) 焊缝区域氢化物分布图

图 2 氢化物金相照片 
的取向与试件内部应力有关。如图 2 所示, 由于未 焊透缝隙尖端的应力集中作用, 氢化物在缝隙尖端 处呈放射状。由于焊缝残余应力的影响, 氢化物在 熔合区靠近热影响区一侧分布更为集中。由图 $2 b$ 和图 2c 可知, 母材区域氢化物的取向相对均匀, 基 本沿水平方向分布, 而焊缝区氢化物表现为混合取 向, 在熔合区左侧, 少量氢化物沿水平向分布, 但 大部分氢化物取向沿坚直方向分布。

如图 3 所示, 选取完全焊透的锆合金焊板, 在 焊缝区域截取拉伸试样完成室温和 $360{ }^{\circ} \mathrm{C}$ 下的拉 伸试验, 试样尺寸如图 4 所示。

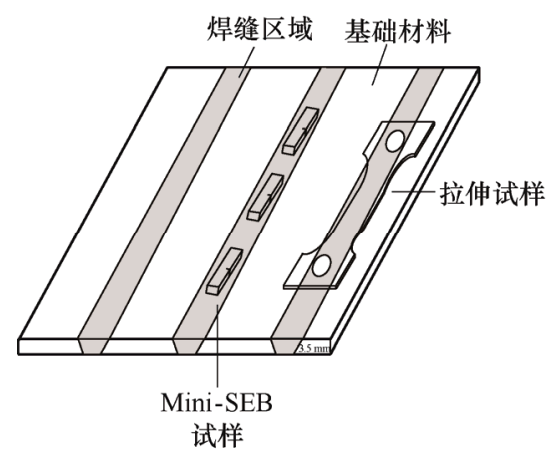

图 3 试样取样示意图

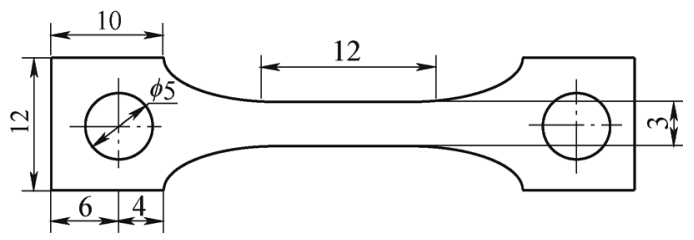

图 4 拉伸试样尺寸示意图

由于锆合金渗氢焊缝的尺寸有限, 无法采用标 准 SEB 或 CT 试样开展断裂性能试验, 故采用图 5 所示的小尺寸 SEB 试样(以下统称为 Mini-SEB 试样) 完成焊缝的断裂性能测试。Mini-SEB 试样的总长度 $L=14 \mathrm{~mm}$, 宽度 $W=3.5 \mathrm{~mm}$, 厚度 $B=3.5 \mathrm{~mm}$, 初始 裂纹长度 $a_{0}$ 可变。热影响区的氢化物分布更加集中, 而对焊缝区的影响较小。因此参照图 3 在焊缝中部 截取试样, 所取试样的裂尖和韧带已离开热影响区, 每条焊缝截取三个 Mini-SEB 试样。由于 Mini-SEB 试样总长度较小, 无法在裂纹嘴位置安装裂尖张开 位移(Crack opening displacement, COD)引伸计, 从 而设计了图 6 所示的加载装置。该加载装置与标准 SEB 试样加载装置类似, 不同之处在于通过刚性外 延的方式测量 Mini-SEB 试样的加载线位移(Loading line displacement, LLD), 试验装置的压头和支撑座 均采用高强高硬合金 GH4169 制成, 测试过程中忽 略压头和支撑座的变形。加载装置与试验机压头和 载荷传感器连接, 压头加载装置与试验机压头和载
荷传感器连接, 压头加载装置与承载台上面分别安 装两个可调节位置的刀口, 测量试样 LLD 的引伸计 安装在刀口上。

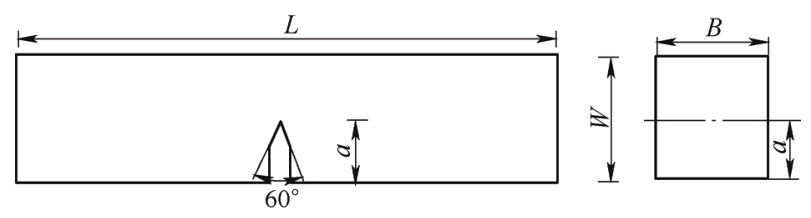

图 5 Mini-SEB 试样尺寸示意图

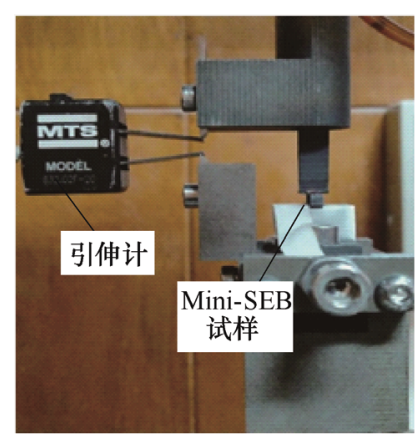

图 6 Mini-SEB 室温试样加载系统

对于高温断裂试验, 采用图 7 所示的加长刚性 外延装置测量试样的加载线位移 LLD。

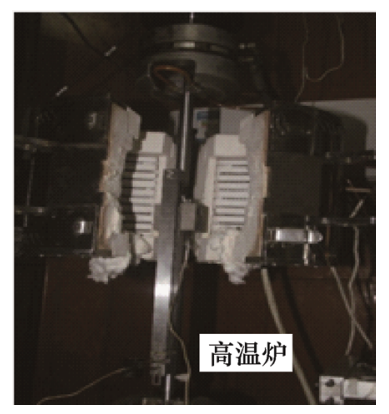

图 7 Mini-SEB 高温试样加载系统

\section{2 研究方法}

\subsection{Mini-SEB 试样应力强度因子 $K$ 的计算}

对于断裂试样的弹性变形行为, CHEN 等 ${ }^{[13-17]}$ 基于能量等效原理, 建立了弹性应变能与载荷的 关系

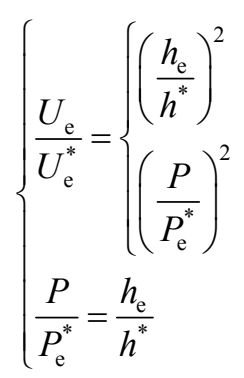

式中, $U_{\mathrm{e}}^{*}=K E V^{*} / 2, P_{\mathrm{e}}^{*}=k E A^{*}, V^{*}=A^{*} h^{*}, E$ 为弹性 
模量, $A^{*}$ 为变形区域特征面积, $h^{*}$ 为特征位移, 取 为试样宽度 $W$ 。

由 $\mathrm{RICE}^{[18]}$ 提出的能量积分表达式

$$
J=-\frac{\partial \Pi}{B \partial a}
$$

式中, $\Pi$ 表示裂纹体总势能, $B$ 表示试样厚度, $a$ 为裂纹长度。可得

$$
J=\sqrt{\frac{P^{2} E^{\prime}}{2 B E k} \frac{\partial}{\partial a}\left(\frac{h^{*}}{A^{*}}\right)}
$$

根据与能量释放率 $G$ 等值的弹性部分 $J$ 积分 $J_{e}$ 与 $K$ 因子的换算公式

$$
G=\frac{K^{2}}{E^{\prime}}
$$

可得 $K$ 为

$$
K=\sqrt{\frac{1}{k} \frac{P^{2} E^{\prime}}{2 B E} \frac{\partial}{\partial a}\left(\frac{h^{*}}{A^{*}}\right)}
$$

令 $h^{*}=W$ 及 $A^{*}=W B(b / W)^{t}$, 将式(5)中的 $E^{\prime}$ 替换为实 际的弹性模量 $E$, 得到 $K$ 的统一公式

$$
K=\frac{P}{B \sqrt{2 W}} \frac{\left[(1 / k) t(b / W)^{t-1}\right]^{0.5}}{(b / W)^{t}}
$$

式中的待定参数仅有 $k 、 t$, 可由线弹性有限元计算 确定。

图 8 为用于 $K$ 计算的三维有限元模型, 有限元 分析借助 ANSYS12.1 进行, 依据试样几何对称性, 有限元模型为 Mini-SEB 试样的结构的 $1 / 2$, 如图 $8 \mathrm{a}$ 所示。

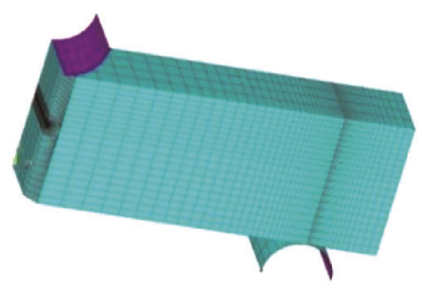

(a) 结构 $1 / 2$ 模型

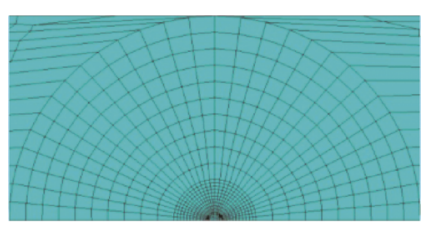

(b) 裂尖网格细化

图 8 Mini-SEB 试样 3D 有限元模型

图 9 给出了相同厚度 $B$ 下不同裂纹试样的载荷位移响应曲线。其斜率为柔度 $C$, 可以看到, 不同 尺寸得到的载荷-位移响应曲线差异明显。

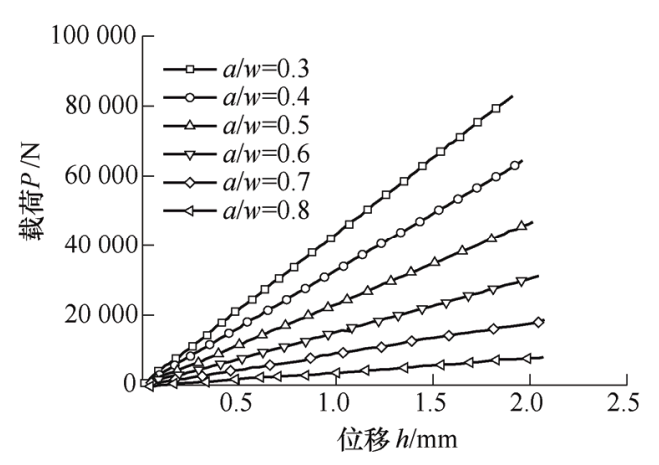

图 9 Mini-SEB 试样的 $P-h$ 曲线

由式(1)可得, Mini-SEB 试样在线弹性条件下的 $P-h_{e}$ 关系为

$$
\frac{P}{E W B\left(\frac{b}{W}\right)^{t}}=k \frac{h}{W}
$$

对式(7)求导可得

$$
1 / C=\frac{\mathrm{d} P}{\mathrm{~d} h}=k E B\left(\frac{b}{W}\right)^{t}
$$

参数 $k$ 与 $t$ 可以由式(8)拟合曲线得出, 表 2 给出了 不同跨距 $S$ 下 Mini-SEB 试样 $K$ 因子算式的待定系 数 $k$ 和 $t$ 。

表 $2 K$ 因子算式待定系数 $\boldsymbol{k}$ 和 $\boldsymbol{t}$

\begin{tabular}{ccc}
\hline$S / W$ & $k$ & $t$ \\
\hline 3 & 0.1418 & 1.875 \\
4 & 0.07582 & 1.835 \\
\hline
\end{tabular}

\subsection{Mini-SEB 试样 $J$ 积分的计算}

BAO 等 ${ }^{[19-20]}$ 研究表明, 试样的载荷 $P$ 可以表示 为 2 个相互独立的几何函数 $g(a / W)$ 和变形函数 $h(V / W)$ 的乘积, 即

$$
\Pi=\frac{P}{\sigma_{0} B W}=g\left(\frac{a}{W}\right) h\left(\frac{V}{W}\right)=\left(1-\frac{a}{W}\right)^{m} h\left(\frac{V}{W}\right)
$$

式中, $\Pi$ 为载荷 $P$ 的无量纲量, $\sigma_{0}$ 为参考屈服应力, $a$ 为裂纹长度, $m$ 为待定参数。

引入分离参数 $S_{i j}$ 来验证式(9)的假定是否成立

$$
S_{i j}=\left.\frac{\Pi_{i}}{\Pi_{j}}\right|_{V / W}=\frac{\left(1-\frac{a_{i}}{W}\right)^{m}}{\left(1-\frac{a_{j}}{W}\right)^{m}}=\left(\frac{b_{j}}{W}\right)^{-m}\left(\frac{b_{i}}{W}\right)^{m}
$$

式中, $\Pi_{i}$ 和 $\Pi_{j}$ 分别为裂纹长度为 $a_{i}$ 和 $a_{j}$ 的针裂纹 试样的无量纲载荷; $b$ 为剩余韧带长度, $b=W-a$ 。 显然, 由于钝裂纹试样在加载过程中不产生裂纹扩 展, 当 $S_{i j}$ 随着位移的增加而保持恒定时, 即可验证 式(9)的成立, 进而, 参数 $m$ 可从 $S_{i j}$ 与 $b_{i} / W$ 的关系 中确定。定义规则化载荷确定实时裂纹长度 


$$
P_{N}=\frac{P}{G(a / W)}=\frac{P}{W B\left(\frac{b}{W}\right)^{\eta}}=H\left(\frac{V}{W}\right)
$$

式中, $P_{N}$ 为规则化载荷。

根据规则化法确定试样加载过程中的实时裂纹 长度后, Mini-SEB 试样的 $J$ 积分采用下列公式计算

$$
J=J_{e}+J_{p}
$$

式中弹性部分

$$
J_{e}=\frac{1-v^{2}}{E} K^{2}
$$

式中, $v$ 为泊松比, $E$ 为等效弹性模量, $K$ 为应力强 度因子。

塑性部分 $J_{p}$ 按增量式计算

$$
J_{p(i)}=\left(J_{p(i-1)}+\frac{\eta}{b_{i-1}} \frac{\Delta U_{p(i)}}{B_{N}}\right) \cdot\left(1-\gamma \frac{a_{(i)}-a_{(i-1)}}{b_{(i-1)}}\right)
$$

式中, $\Delta U_{p}$ 塑性功增量

$$
\Delta U_{p(i)}=\frac{\left(P_{(i)}+P_{(i-1)}\right)\left(V_{p(i)}-V_{p(i-1)}\right)}{2}
$$

式中, $W 、 B_{N} 、 a$ 和 $b$ 分别为试样宽度、试样净厚 度、裂纹长度和剩余韧带长, $\eta$ 和 $\gamma$ 为与试样几何 构形相关的塑性因子。由于本文研究所用 Mini-SEB 试样为标准 SEB 试样等比例缩小后的试样故可取塑 性因子 $\eta$ 为固定值 1.9 , 而 $\gamma$ 可取为 0.9 。

\section{3 结果与分析}

室温和 $360{ }^{\circ} \mathrm{C}$ 下的拉伸试验, 结果如表 3 所示。 可以看到, 不同工况下的拉伸试验结果存在少许分

\begin{tabular}{|c|c|c|c|c|c|}
\hline 工况 & 温度 & 试样编号 & $\begin{array}{c}\text { 弹性模量 } \\
E / \mathrm{MPa} \\
\end{array}$ & $\begin{array}{l}\text { 抗拉强度 } \\
\sigma_{\mathrm{p} 0.2} / \mathrm{MPa} \\
\end{array}$ & $\begin{array}{c}\text { 屈服强度 } \\
\sigma_{\mathrm{b}} / \mathrm{MPa} \\
\end{array}$ \\
\hline \multirow{3}{*}{ 未渗氢 } & \multirow{3}{*}{ 室温 } & weld-rt-1\# & 88426 & 580 & 606 \\
\hline & & weld-rt-2\# & 94639 & 575 & 599 \\
\hline & & weld-rt-3\# & 91601 & 574 & 601 \\
\hline \multirow{6}{*}{ 渗氢 } & \multirow{3}{*}{ 室温 } & weld-rt-1\# & 89851 & 424 & 527 \\
\hline & & weld-rt-2\# & 85703 & 416 & 510 \\
\hline & & weld-rt-3\# & 92693 & 457 & 558 \\
\hline & \multirow{3}{*}{$360{ }^{\circ} \mathrm{C}$} & weld-360-1\# & 60345 & 227 & 313 \\
\hline & & weld-360-2\# & 74748 & 227 & 304 \\
\hline & & weld-360-3\# & 57268 & 237 & 322 \\
\hline
\end{tabular}
散性。室温下, 渗氢后焊缝的弹性模量变化不大, 但屈服强度和抗拉强度明显降低。对于渗氢焊缝, $360{ }^{\circ} \mathrm{C}$ 下的拉伸结果明显低于室温结果。

表 3 锆合金焊缝室温与 $360{ }^{\circ} \mathrm{C}$ 拉伸性能

图 10 给出了未渗氢焊缝室温下 5 个试样的载荷 $P$ 与加载线位移曲线。图 11 给出对应的 $J$ 阻力曲线。

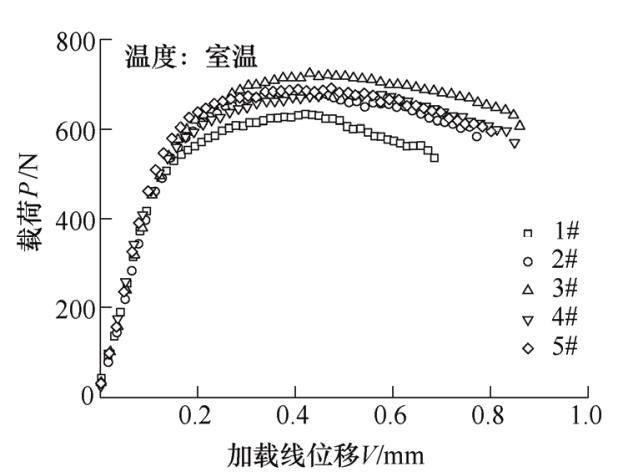

图 10 未渗氢焊缝室温下的 $P-V$ 曲线

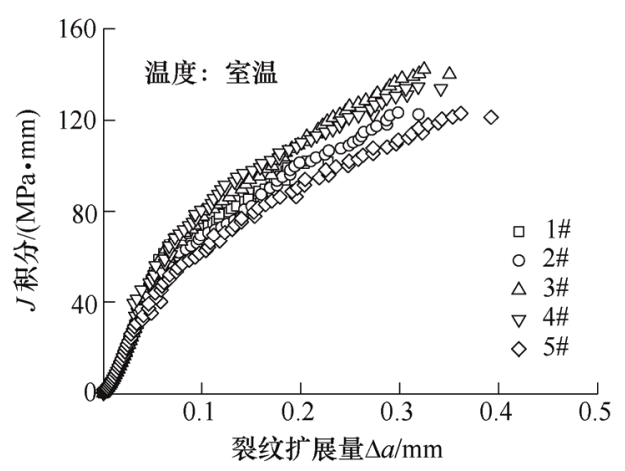

图 11 未渗氢焊缝室温下的 $J-\Delta a$ 阻力曲线

图 12 给出了渗氢焊缝室温下 10 个试样的载荷 $P$ 与加载线位移曲线。图 13 给出对应的 $J$ 阻力曲线。 可以看到, 渗氢焊缝试样室温下的 $P-V$ 曲线和 $J$ 阻 力曲线均存在一定的分散性。

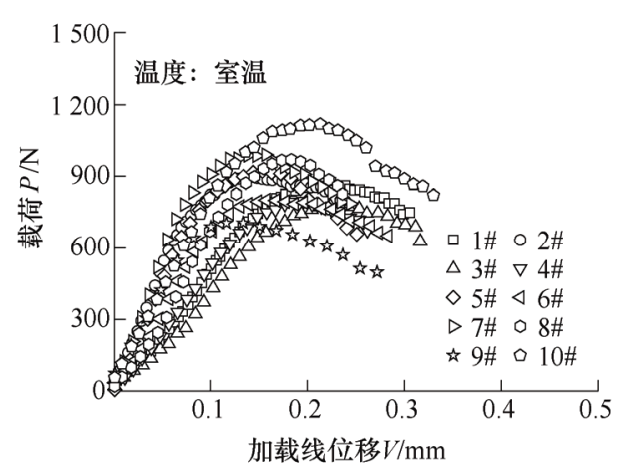

图 12 渗氢焊缝室温下的 $P-V$ 曲线

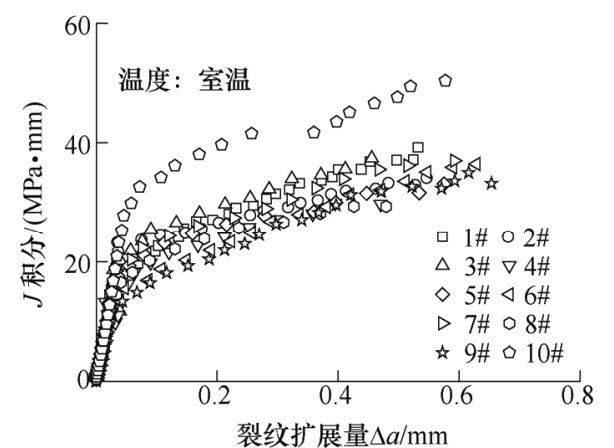

图 13 渗氢焊缝室温下的 $J-\Delta a$ 阻力曲线 
图 14 给出了渗氢焊缝 $360{ }^{\circ} \mathrm{C}$ 下 10 个试样的载 荷 $P$ 与加载线位移曲线。图 15 给出对应的 $J$ 阻力曲 线。可以看到, 渗氢焊缝试样 $360{ }^{\circ} \mathrm{C}$ 下的 $P-V$ 曲线、 $J$ 阻力曲线均存在分散性。

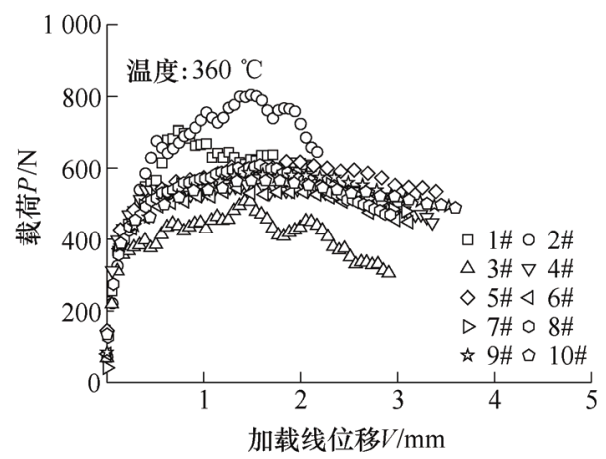

图 14 渗氢焊缝 $360{ }^{\circ} \mathrm{C}$ 下的 $P-V$ 曲线

表 4 列出了未渗氢焊缝室温下的断裂韧度结果
渗氢焊缝室温与 $360{ }^{\circ} \mathrm{C}$ 下的断裂韧度结果。

从表 4 的断裂韧度结果还可以看到, 室温下, 渗氢焊缝的断裂韧度因氢化物的影响, 显著下 降, 低于未渗氢焊缝的断裂韧度。这是由于室温 下聚集在裂纹尖端附近的脆性氢化物导致试样 在低应力条件下启裂扩展, 从而表现出较低的断 裂韧度。360 ${ }^{\circ} \mathrm{C}$ 下渗氢焊缝的断裂韧度明显高于 室温下未渗氢和渗氢焊缝的断裂韧度。其原因在 于, 一方面, 试验温度升高必将使焊缝材料的延 性增强, 断裂韧度提高; 另一方面，锆合金焊缝 在高温腐蚀条件下吸氢, 当冷却室温时, 便以脆 性氢化物的形式析出; 当渗氢锆合金焊缝断裂试 样再次处于高温环境下时, 聚集在裂纹尖端附近 的脆性氢化物将部分溶解或扩散, 从而表现出比 室温下更好的延性。

表 4 室温与 $360{ }^{\circ} \mathrm{C}$ 下渗氢焊缝断裂韧性结果

\begin{tabular}{|c|c|c|c|c|c|c|}
\hline 工况 & 温度 & 试样编号 & $a_{0} / W$ & 裂纹扩展量 $\Delta a_{\max } / \mathrm{mm}$ & 裂纹总长 $a_{\mathrm{f}} / \mathrm{mm}$ & 断裂㓞度 $J_{Q} /(\mathrm{MPa} \cdot \mathrm{mm})$ \\
\hline \multirow{5}{*}{ 未渗氢 } & \multirow{5}{*}{ 室温 } & $1 \#$ & 0.510 & 0.228 & 2.115 & 119 \\
\hline & & $2 \#$ & 0.519 & 0.319 & 2.238 & 123 \\
\hline & & $3 \#$ & 0.500 & 0.351 & 2.201 & 142 \\
\hline & & $4 \#$ & 0.509 & 0.342 & 2.225 & 134 \\
\hline & & $5 \#$ & 0.479 & 0.393 & 2.164 & 108 \\
\hline \multirow{20}{*}{ 渗氢 } & \multirow{10}{*}{ 室温 } & $1 \#$ & 0.391 & 0.599 & 2.045 & 29.4 \\
\hline & & $2 \#$ & 0.373 & 0.595 & 1.975 & 26.9 \\
\hline & & $3 \#$ & 0.431 & 0.560 & 2.154 & 26.5 \\
\hline & & $4 \#$ & 0.381 & 0.562 & 1.971 & 25.2 \\
\hline & & $5 \#$ & 0.356 & 0.720 & 2.039 & 30.9 \\
\hline & & $6 \#$ & 0.409 & 0.630 & 2.144 & 24.9 \\
\hline & & $7 \#$ & 0.424 & 0.603 & 2.171 & 22.6 \\
\hline & & $8 \#$ & 0.369 & 0.605 & 1.969 & 19.7 \\
\hline & & $9 \#$ & 0.354 & 0.698 & 2.007 & 21.2 \\
\hline & & $10 \#$ & 0.385 & 0.556 & 1.980 & 20.4 \\
\hline & \multirow{10}{*}{$360{ }^{\circ} \mathrm{C}$} & $1 \#$ & 0.372 & 0.352 & 1.728 & 228 \\
\hline & & $2 \#$ & 0.341 & 0.440 & 1.700 & 235 \\
\hline & & $3 \#$ & 0.371 & 0.642 & 2.016 & 228 \\
\hline & & $4 \#$ & 0.390 & 0.736 & 2.178 & 185 \\
\hline & & $5 \#$ & 0.380 & 0.985 & 2.390 & 148 \\
\hline & & $6 \#$ & 0.404 & 0.550 & 2.043 & 182 \\
\hline & & $7 \#$ & 0.380 & 0.678 & 2.084 & 216 \\
\hline & & $8 \#$ & 0.386 & 0.831 & 2.257 & 188 \\
\hline & & $9 \#$ & 0.391 & 0.843 & 2.288 & 183 \\
\hline & & $10 \#$ & 0.387 & 0.843 & 2.275 & 188 \\
\hline
\end{tabular}


从图 11 可以看到, 虽然各试样的初始裂纹尺寸 有差异, 但各试样的 $J$ 阻力曲线分散性相对较小, 表明未渗氢条件下, 焊缝断裂韧性受初始裂纹尺寸 的影响较小。

从图 13 和图 15 可以看出, 同为渗氢焊缝, 室 温下的 $J$ 阻力曲线相比 $360{ }^{\circ} \mathrm{C}$ 下的 $J$ 阻力曲线更加 分散。其原因为室温下断裂试样裂纹尖端附近聚集 的脆性氢化物导致试样低应力启裂扩展, 而氢化物 的不均匀分布增加了其断裂的不确定性。360 ${ }^{\circ} \mathrm{C}$ 下, 聚集在裂尖附近的氢化物部分溶解或扩散, 不仅提 升了渗氢焊缝的断裂韧度也减小了其断裂不确定 性, 从而表现出更小的分散性。

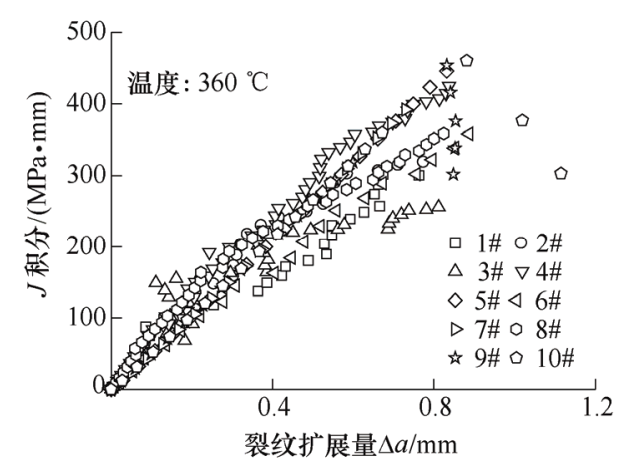

图 15 渗氢焊缝 $360{ }^{\circ} \mathrm{C}$ 下的 $J-\Delta a$ 阻力曲线

\section{4 结论}

（1）基于弹塑性有限元分析建立了 Mini-SEB 试 样的 $K$ 因子表达式、 $J$ 积分算式。

（2）室温下, 渗氢焊缝由于裂纹尖端附近的脆 性氢化物聚集导致其在低应力条件下启裂扩展, 相 对于未渗氢焊缝的断裂韧度明显下降; 由于氢化物 非均匀分布导致渗氢焊缝在室温下的 $J$ 阻力曲线存 在明显的分散性。

（3）高温下, 聚集在裂纹尖端附近的脆性氢化 物部分溶解或扩散, 不再具有脆性特征, 使得渗氢 焊缝在高温下的断裂㓞度较室温下的断裂韧度明显 提升, 分散性减小。

\section{参 考 文 献}

[1] 杨芳林, 张建军, 宋启忠. 锆合金管材在氢氧化锂溶液 中的吸氢研究[J]. 稀有金属, 1999, 19(3): 58-63.

YANG Fanglin, ZHANG Jianjun, SONG Qizhong. Study on hydrogen absorption of Zirconium tubes in lithum hydroxide solution[J]. Rare Metal, 1999, 19(3): 58-63.

[2] 唐睿, 杨晓雪. N18、Zry-4 和 M5 锆合金中氢的固溶 度[J]. 材料研究学报, 2009, 23(6): 77-81.
TANG Rui, YANG Xiaoxue. Solid solubility of hydrogen in N18, Zry-4 and M5 zirconium alloys[J]. Chinese Journal of Materials Research, 2009, 23(6): 77-81.

[3] STEINBRUECK M, VESHCHUNOV M S, BOLDYREV $\mathrm{A} \mathrm{V}$, et al. Oxidation of $\mathrm{B}_{4} \mathrm{C}$ by steam at high temperatures: New experiments and modelling[J]. Nuclear Engineering and Design, 2007, 237(2): 161-181.

[4] 周军, 李中奎, 张建军, 等. 不同氢含量的新锆合 金(NZ2)的力学性能 [J]. 稀有金属材料与工程, 2008 (12): 179-183.

ZHOU Jun, LI Zhongkui, ZHANG Jianjun, et al Mechanical properties of new zirconium alloy(N22) with different hydrogen content[J]. Rare Metal Materials and Engineering, 2008(12): 179-183.

[5] 王瑞红, 丁向东, 肖林, 等. 渗氢前后 $\mathrm{Zr}-4$ 合金板的力 学性能及有限元模拟 $[\mathrm{J}]$. 中国有色金属学报, 2002 , 12(3): 544-549.

WANG Ruihong, DING Xiangdong, XIAO Lin, et al. Mechanical properties and finite simulation of $\mathrm{Zr}-4$ alloy plate before and after hydrogenation[J]. Transaction of Nonferrous Metals Society of China, 2002, 12(3): 544-549.

[6] 王瑞红, 丁向东, 刘刚, 等. 氢化物在 Zr-4 合金中变形 行为的研究 $[\mathrm{J}]$. 稀有金属材料与工程, 2003, 6(12): 156-160.

WANG Ruihong, DING Xiangdong, LIU Gang, et al. Numerical analysis of deformation behavior of $\mathrm{Zr}-4$ alloy[J]. Rare Metal Materials and Engineering, 2003, 6(12): 156-160.

[7] 刘肖, 王理, 包陈, 等. 含轴向对称裂纹锆合金包壳管 断裂行为 $[\mathrm{J}]$. 机械工程学报, 2019, 55(16): 76-85.

LIU Xiao, WANG Li, BAO Chen, et al. Study on fracture behaviour of zirconium alloy cladding tubes containing axial symmetric cracks[J]. Journal of Mechanical Engineering, 2019, 55(16): 76-85.

[8] 贾琦, 蔡力勋, 包陈, 等. 锆合金薄壁细管的单调拉伸 与低周疲劳试验研究 $[\mathrm{J}]$. 原子能科学技术, 2010, 44(6): 712-717.

JIA Qi, CAI Lixun, BAO Chen, et al. Monotonic tensile and low cycle fatigue tests of zirconium thin walled tubes[J]. Atomic Technology, 2010，44(6): 712-717.

[9] 孙超, 谭军, 应诗浩, 等. N18 锆合金氢致裂纹延迟开 裂临界温度研究 $[J]$. 金属学报, 2009, 45(5): 541-546. SUN Chao, TAN Jun, YING Shihao, et al. Study on the critical temperature of delayed crack induced by hydrogen in N18 zirconium alloys[J]. Acta Metallurgica Sinica, 
2009, 45(5): 541-546.

[10] HSU H H, TSAY L W. Effect of hydride orientation on fracture toughness of Zircaloy-4 cladding[J]. Journal of Nuclear Materials, 2011, 408(1): 67-72.

[11] MIN S J, KIM M S, KIM K T. Cooling rate and hydrogen content-dependent hydride reorientation and mechanical property degradation of $\mathrm{Zr}-\mathrm{Nb}$ alloy claddings[J]. Journal of Nuclear Materials， 2013， 441(1-3): 306-314.

[12] TSENG C C, SUN M H, CHAO C K. Hydride effect on crack instability of zircaloy cladding[J]. Nuclear Engineering and Design, 2014, 270: 427-435.

[13] CHEN Hui, CAI Lixun. Theoretical model for predicting uniaxial stress-strain relation by dual conical indentation based on equivalent energy principle[J]. Acta Materialia, 2016, 121: 181-189.

[14] CHEN Hui, CAI Lixun. Unified elastoplastic model based on strain energy equivalence principle[J]. Applied Mathematical Modelling, 2017, 52: 664-671.

[15] CHEN Hui, CAI Lixun. Unified ring-compression model for determining tensile properties of tubular materials[J]. Materials Today Communication, 2017, 13: 210-220.

[16] CHEN Hui, CAI Lixun. Theoretical conversions of different hardness and tensile strength for ductile materials based on stress-strain curves $[\mathrm{J}]$. Metallurgical and Materials Transactions A, 2018, 49(4): 1090-1101.
[17] 贺屹, 蔡力勋, 陈辉. 基于能量等效原理的 $\mathrm{K}$ 因子、柔 度统一模型 $[\mathrm{J}]$. 机械工程学报, 2018，54(14): 98-106. HE Yi, CAI Lixun, CHEN Hui. Unified model of K factor and compliance based on energy equivalence principle $[\mathrm{J}]$. Journal of Mechanical Engineering, 2018, 54(14): 98-106.

[18] RICE J R. A path independent integral and the approximate analysis of strain concentration by notches and cracks[J]. Journal of Applied Mechanics, 1968, 35(2): 379-386.

[19] BAO Chen, CAI Lixun. Normalization method for evaluating J-resistance curves of small-sized CIET specimen and crack front constraints[J]. International Journal of Solids and Structures, 2016, 94: 60-75.

[20] BAO Chen, CAI Lixun, SHI Kaikai, et al. Improved normalization method for ductile fracture toughness determination based on dimensionless load separation principle[J]. Acta Mechanical Solid Sinica, 2015, 28(2): 168-181.

作者简介: 王博, 男, 1994 年出生。主要研究方向为材料断裂行为。 E-mail: wyb54635593@163.com

包陈, 男, 1982 年出生, 副教授。主要研究方向为结构完整性评价。 E-mail: bchxx@163.com

魏连峰, 男, 1981 年出生, 硕士, 助理研究员。主要研究方向为核燃料 与材料焊接技术及工程。

E-mail:252044665@qq.com

何广伟, 男, 1991 年出生, 博士研究生。主要研究方向为材料断裂行为。 E-mail:619041277@qq.com 